\title{
Near-field imaging for single-shot waveform measurements
}

\author{
TJ Hammond ${ }^{1,2,3}$, Aleksey Korobenko $^{1,2}$, A Yu Naumov ${ }^{1,4}$, \\ D M Villeneuve ${ }^{1,4} \oplus$, Paul B Corkum ${ }^{1,2}$ and Dong Hyuk Ko ${ }^{1,2}$ \\ ${ }^{1}$ Joint Attosecond Science Laboratory, National Research Council of Canada and University of Ottawa, \\ 100 Sussex Dr, Ottawa, ON K1A 0R6, Canada \\ ${ }^{2}$ Department of Physics, University of Ottawa, 25 Templeton St, Ottawa, ON K1N 6N5, Canada \\ ${ }^{3}$ Institute for the Frontier of Attosecond Science and Technology, CREOL and Department of Physics, \\ University of Central Florida, Orlando, FL 32816, United States of America \\ ${ }^{4}$ National Research Council of Canada, 100 Sussex Dr, Ottawa, ON K1A 0R6, Canada \\ E-mail: thammond@uottawa.ca
}

Received 12 December 2017, revised 12 January 2018

Accepted for publication 9 February 2018

Published 28 February 2018

\begin{abstract}
The accurate measurement of ultrashort pulses is necessary to optimize pulse compression for nonlinear optics and strong-field physics. Additionally, field-sensitive time-domain techniques enable measuring femtosecond and sub-femtosecond dynamics. In this paper, we introduce a single-shot measurement to characterize few-cycle fields. This polarization-sensitive timedomain measurement can retrieve the waveform of pulses shorter than two-cycles in duration with a temporal window of hundreds of femtoseconds. We measure how the field changes after propagation through dispersive optics, and we measure the sub-femtosecond phase shift imposed by the nonlinear intensity dependent response of a dielectric. Real-time field measurements will benefit low repetition rate systems used in strong-field physics and attosecond science, while the large temporal window compliments Fourier transform spectroscopy.
\end{abstract}

Supplementary material for this article is available online

Keywords: waveform measurement, ultrafast measurements, ultrafast nonlinear optics, pulse characterization

(Some figures may appear in colour only in the online journal)

\section{Introduction}

The precise measurement and control of visible and near infrared fields over a broad spectral bandwidth enables coherent control experiments [1, 2]. Time-domain measurements such as transient absorption [3-5] and Fourier transform infrared (FTIR) spectroscopy $[6,7]$ benefit from a broad temporal range, with sub-femtosecond resolution and picosecond windows. High harmonic and attosecond pulse generation require the accurate measurement of the amplitude and phase of few-cycle driving fields in order to optimize the generated yield [8] and maximum photon energy $[9,10]$. Fortunately, attosecond technologies established pulse characterizing techniques that-while simultaneously characterizing the attosecond pulse-led to the accurate measurement of few-cycle electric fields [11]: an ex situ measurement from photoelectron streaking $[12,13]$, and an in situ probing of the attosecond pulse generation itself [14]. However, these techniques are time consuming and we are interested in measurements that can be performed in a single shot [15].

In this paper, we propose and demonstrate a time-domain measurement of an electric field in a single shot while avoiding the use of extreme ultraviolet (XUV) optics and vacuum chambers required for isolated attosecond pulse characterization. To this end, we interfere a stronger fewcycle pulse (the 'generating beam' with central wavelength of $1.8 \mu \mathrm{m}$ ) with a weaker perturbing beam (the 'signal field') during third harmonic generation. We observe the near-field modulation the signal field imposes on the generating beam Gaussian profile to measure the signal field's temporal character. In contrast to other pulse characterizing techniques, the near-field distribution allows us to directly observe the signal field and simplifies the interpretation. 
The third harmonic of the generating beam is in the visible spectrum and it is easily magnified by silver mirrors and imaged by a charge couple device (CCD) camera. By magnifying and observing the near-field interference of the third harmonic generation process, we accurately measure the signal electric field up to a constant phase. Unlike attosecond field-sensitive techniques, we show that the measurement is nearly insensitive to the temporal profile of the generating beam. Furthermore, unlike auto-correlation measurements, our technique does not require any assumptions about the pulse envelope and can directly detect any temporal phase change within the pulse. We show that while this single-shot technique has a temporal window of several hundred femtoseconds, it also has sub-cycle resolution.

The wide temporal window is necessary for measuring pre- and post-pulses and plateaux that accompany few-cycle pulses, or more complicated pulse shapes used for coherent control. Because this time-domain measurement is sensitive to the polarization, we easily measure the rapidly varying polarization of a few-cycle pulse used for polarization gating to generate an isolated attosecond pulse [16, 17]. We also measure the nonlinear phase delay caused by the intensity dependent phase shift in material, accurately measuring the induced sub-femtosecond nonlinear light-matter interaction.

\section{Methods}

We focus the carrier-envelope phase stable idler of an optical parametric amplifier (OPA) (light conversion HE-TOPAS), central wavelength of $1.8 \mu \mathrm{m}$, onto a hollow-core fibre filled with argon at 1.1 bar to generate a supercontinuum spectrum supporting two-cycle pulses. A $0.5 \mathrm{~mm}$ fused silica plate separates the collimated S-polarized beam into two arms, splitting off $20 \mu \mathrm{J}$ for the signal field from the $300 \mu \mathrm{J}$ used for the generating beam. We further reduce the signal field power with an iris, which ensures that the signal field is only weakly perturbing and has a larger spot size than the generating beam profile. We focus the two beams using two $1 \mathrm{~m}$ focal length mirrors, relative angle of $\theta=200 \mathrm{mrad}$, onto a thin $(100 \mu \mathrm{m}$ thick) quartz plate. A schematic of the measurement is shown in figure 1; here we show a strong interference for illustrative purposes. Further experimental details are provided in the supplemental information is available online at stacks.iop. org/JPB/51/065603/mmedia.

When the signal field spatially and temporally overlaps with the generating beam within the quartz plate, the crosscorrelation signal gives four distinct third harmonic beams, labelled 1-4. These four beams consist of the four possible third harmonic photon configurations: three generating, two generating and one signal, one generating and two signal, and three signal photons. The fundamental field is removed via a series of dichroic mirrors that reflect $1.8 \mu \mathrm{m}$ wavelength. We collect all four third harmonic beams to magnify this interaction $10 \times$ via a $10 \mathrm{~cm}$ focal length silver mirror onto a CCD camera. Because the signal field is larger in the near-field than

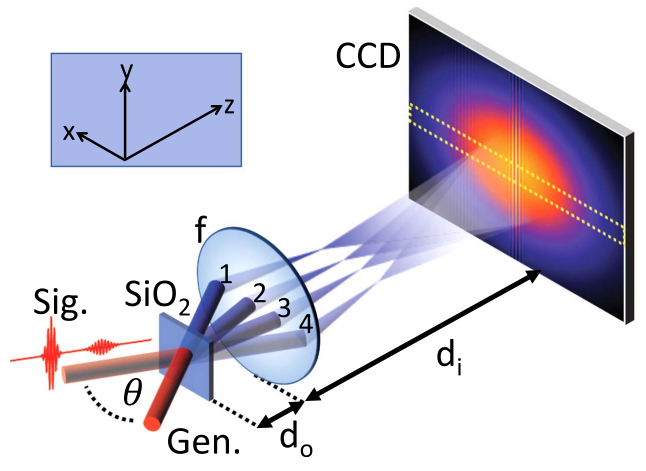

Figure 1. Schematic of the measurement. The signal field (sig.) perturbs the generating beam (gen.) within the thin $\mathrm{SiO}_{2}$ to produce four diffracted third harmonic beams. We magnify and image this interaction with a mirror (lens in figure f) on a CCD (false colour image) to characterize the signal electric field via the interference fringes. Inset: Cartesian coordinates.

the generating beam, the modulation depth is not uniform in the vertical plane (the $y$ axis). To maintain the weak modulation for the measurement, only the profile within the dashed yellow box yields the measured interference.

The total temporal window, $T$, is

$$
T=\frac{\theta}{M c} W
$$

where $W$ is the detector size, $M=d_{i} / d_{o}$ is the magnification, and $c$ is the speed of light. From our CCD camera having 640 pixels with a $5 \mathrm{~mm}$ wide sensor gives us a temporal window of $330 \mathrm{fs}$. In practice, we can consistently characterize the signal field within the waist of the generating beam; in our case the practical temporal window is approximately $200 \mathrm{fs}$.

\section{Theory}

Pulse characterization often requires splitting the test pulse into two equal amplitudes and recombining them on some nonlinear medium [18-20]. We argue that modifying the third harmonic generation from a strong generating beam with a weak perturbing field can fully temporally characterize the perturbing field (up to a constant phase). The generating and signal fields, respectively, are

$$
\begin{aligned}
& G(x, z, t)=G\left(t-\frac{z}{c} \cos \theta_{1}+\frac{x}{c} \sin \theta_{1}\right), \\
& S(x, z, t)=S\left(t-\frac{z}{c} \cos \theta_{2}-\frac{x}{c} \sin \theta_{2}\right),
\end{aligned}
$$

where $\theta_{1}, \theta_{2}$ are the angles of incidence for the generating and signal beams, respectively. For the symmetric geometry where $\theta_{1}=\theta_{2}=\theta / 2$, then we obtain a temporal delay

$$
\tau=t-\frac{2 x}{M c} \sin (\theta / 2) \approx t-\frac{\theta}{M c} x,
$$

for $\theta \ll 1$. In this geometry there are no geometrical distortions caused by the propagation $z$ [19]. When the two pulses overlap, we can set $t=0$ and map the $x$ coordinate of the camera to a delay $\tau$. 
The resulting third harmonic signal caused by a weak perturbation of our signal field $S(x)$ on the generating beam $G$ $(x)$ is

$$
I_{3 \omega}(x)=\left|\int_{0}^{L}[G(x, z)+S(x, z)]^{3} \mathrm{~d} z\right|^{2} .
$$

Assuming that the interaction is phase matched over length $L$, then

$$
\begin{aligned}
I_{3 \omega}(x) / L^{2}= & |G(x)+S(x)|^{6} \\
= & |G(x)|^{6}+6|G(x)|^{5}|S(x)| \cos \Delta \phi(x) \\
& +3|G(x)|^{4}|S(x)|^{2}[3+2 \cos 2 \Delta \phi(x)]+\ldots,
\end{aligned}
$$

where $\Delta \phi(x)=\frac{\theta \omega}{M c} x$ is the phase difference of the generating and signal fields at angular frequency $\omega$ and gives rise to the interference.

The first term, $|G(x)|^{6}$, does not contain the perturbation. We assume that this term is simply the generating beam shape and can be easily verified. By taking the Fourier transform of $I_{3 \omega}(x)$, we can isolate and remove this component. In the case that $|S(x)| \ll|G(x)|$, then we are limited to the first two terms in equation (4) with the result being the signal field. That is,

$$
S(\tau)=|S(x)| \cos \Delta \phi(x)=\frac{I_{3 \omega}(x) / L^{2}-|G(x)|^{6}}{6|G(x)|^{5}} .
$$

We demonstrate how we obtain this signal field in practice in figure 2. The horizontal lineout of the recorded image shows the generating beam near-field Gaussian spatial profile, $|G(x)|^{6}$, with a small modulation caused by the weak signal field shown in (a). We map the position coordinate $x$ to the time delay $\tau$. We take the Fourier transform to observe the spectrum, now in units of $1 / \tau$. The Gaussian beam shape background becomes the low frequency (LF) component (pink), while the higher frequency (HF) component (green) contains the signal. We separate these two spectral components and we Fourier transform back to the temporal domain, easily distinguishing the profile of the generating beam and the interference caused by the signal field. Finally, we account for the beam shape by dividing the HF signal by $\mathrm{LF}^{5 / 6}$ to reconstruct $S(\tau)$, where the $5 / 6$ power is in the denominator of equation (5).

This single-shot application for laser monitoring by optical nonlinearity (SALMON) is a robust technique for measuring few-cycle laser pulses in the time domain. Because of the third-order nonlinearity involved, we can accurately reconstruct the signal field even when the generating pulse is longer than the signal field, and we are insensitive to satellite pulses in the generating beam. Additionally, the modulation depth is proportional to the electric field amplitude of the signal (and not the intensity), which allows us to measure very weak fields.

Furthermore, it is apparent when the signal field relative intensity is too high because the next order term in equation (4) modulates at twice the frequency. Simultaneously observing the signal in the time domain and computing the spectrum allows us to ensure that we remain within
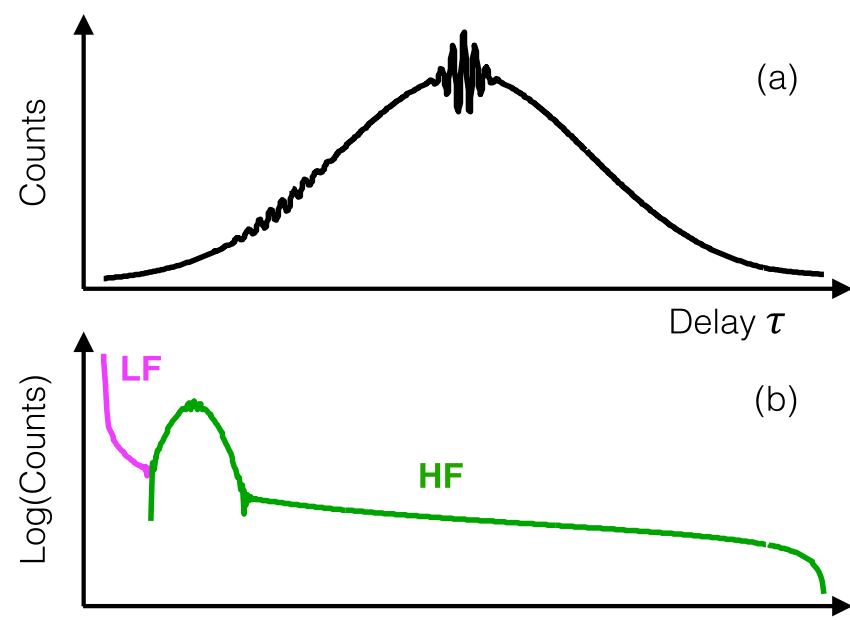

$1 / \tau$
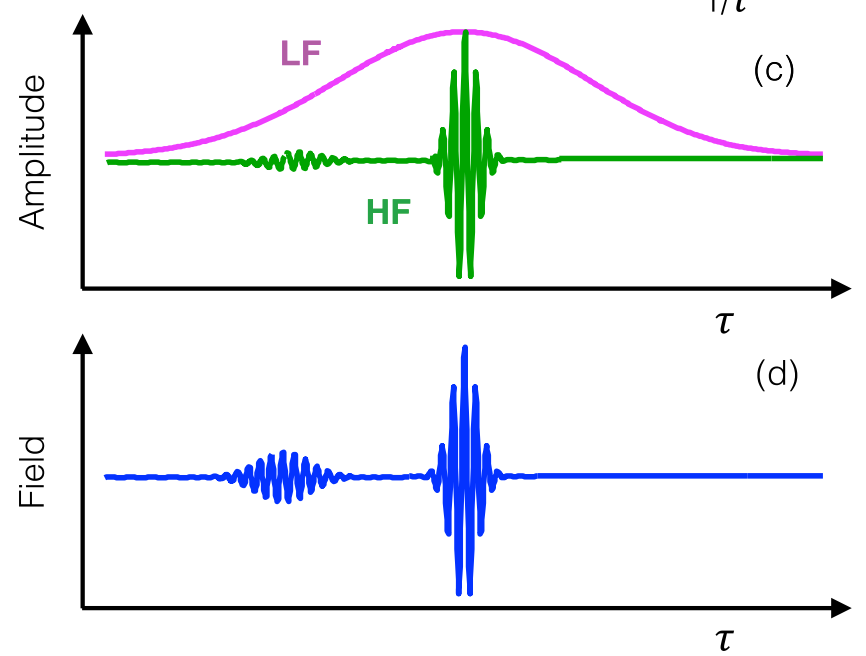

Figure 2. The algorithm for reconstructing the signal field $S(\tau)$. (a) The lineout (or sum over small region) of the CCD in the $x$ coordinate shows the near-field image of the broad Gaussian generating beam shape with an interfering component; we can map $x$ to a relative delay $\tau$. (b) We Fourier transform the distribution to separate the low and high frequency (LF pink, HF green) components. Inverse Fourier transforming back separates the first and second terms from equation (4). Accounting for LF yields $S(\tau)$ over the temporal window, showing the two-cycle main pulse and properly accounts for the amplitude of the weak pre-pulse.

the perturbative regime. We can satisfy the requirement when the computed second harmonic portion of the spectrum is several orders of magnitude lower than the fundamental, typically when $|S| /|G| \sim 1 \%$. In this case, the modulation amplitude is greater than $5 \%$. However, we can reduce this constraint and increase the signal field relative amplitude if we also filter out the computed second harmonic, imposing a broad bandpass filter about the fundamental frequency.

As stated previously, $\Delta \phi(x)$ is the phase difference of the generating beam and signal field. The global phase for a few-cycle pulse is determined by the carrier-envelope phase (CEP) of the laser pulse and so this technique cannot measure the absolute phase directly. However, it is possible to measure the relative CEP of the signal field to the generating beam. Additionally, we cannot measure fields that contain the third harmonic. For example, the argon-filled hollow-core fibre that 

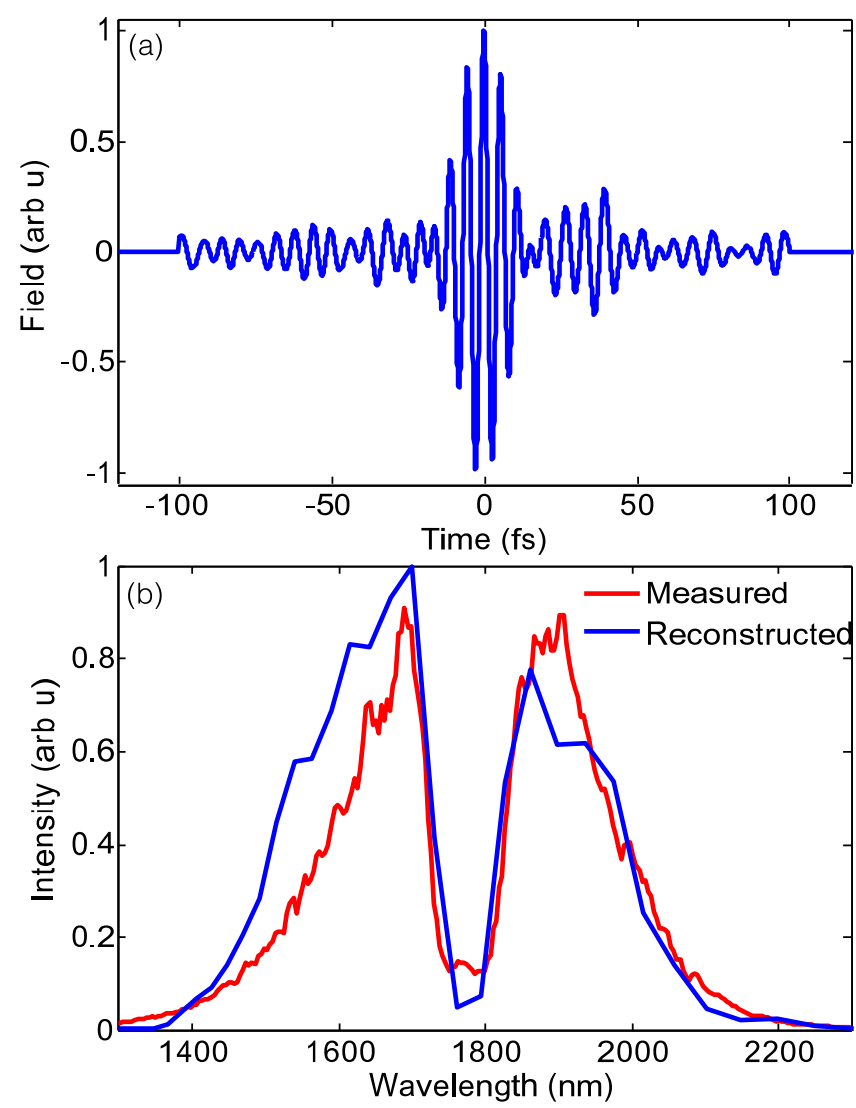

Figure 3. (a) The single-shot time-domain field measurement of the two-cycle pulse. (b) Spectra measured with spectrometer (red) and reconstructed from the measured field (blue).

we use to generate the two-cycle pulse also simultaneously creates third harmonic. This third harmonic must be removed before generating $I_{3 \omega}$ because it will interfere with the signal at the CCD.

\section{Results}

We measure the electric field of a two-cycle pulse via the SALMON method in several different cases. First, we measure the signal field when both the generating beam and the signal field are near the Fourier transform. We compare this measurement to an attosecond field-sensitive technique, the $\mathrm{PHz}$ optical oscilloscope. We test the dispersion sensitivity of the field measurement with respect to the generating beam and the signal field. We also show that this measurement is insensitive to the relative delay, but is sensitive to the relative phase of the generating and signal fields. Finally, we show that we are able to resolve sub-femtosecond dynamics induced in intensity dependent processes.

\subsection{Field measurement and spectrum}

We begin by showing a near Fourier transform limited pulse in figure 3(a). In this case, we have compressed the signal field to the minimum pulse duration while the generating beam is also near transform limited. We compare the measured fundamental spectrum (red) with the retrieved spectrum (blue) in (b). The $200 \mathrm{fs}$ window allows for $50 \mathrm{~nm}$ spectral resolution at $1.8 \mu \mathrm{m}$.

\subsection{PHz optical oscilloscope comparison}

The PHz optical oscilloscope [21] measures the electric field of a signal arm by deflecting the XUV continuum of an isolated attosecond pulse. The setup for this measurement is shown in figure 4(a). The driving field is the CEP stabilized $1.8 \mu \mathrm{m}$ idler from an OPA compressed to sub-two-cycles.

We briefly describe the $\mathrm{PHz}$ optical oscilloscope technique setup here. The short pulse is split into two arms using a $0.5 \mathrm{~mm}$ fused silica plate, with $8 \%$ reflection (S polarized) going to the signal arm. The generating arm passes through polarization gating optics $(200 \mu \mathrm{m}$ thick quartz plate, with optic axis rotated $45^{\circ}$ relative to the laser polarization, and a $\lambda / 4$ wave plate). The $\lambda$ delay imposed by the quartz plate preserves the S polarization in the XUV. We optimize the pulse compression using the anomalous dispersion of fused silica wedges (not shown). A $30 \mathrm{~cm}$ focal length $\mathrm{Ag}$ mirror focusses the generating pulse to a peak intensity of $1 \times 10^{14} \mathrm{~W} \mathrm{~cm}^{-2}$, and we use a gas jet with $\mathrm{Kr}$ (backing pressure 4 bar) to generate an XUV continuum up to $100 \mathrm{eV}$. We detect the XUV with a $2 \mathrm{D}$ spectrometer that measures from 20 to $80 \mathrm{eV}$, and we take the portion of the spectrum around $25 \mathrm{eV}$ to measure the signal field [22].

We control the dispersion of the signal arm independent of the generating arm with thin fused silica plates and optimize the signal field pulse compression. We delay the signal field using a computer controlled PZT delay stage with nm resolution, with 250 as steps. The integration time is approximately $1 \mathrm{~s}$ per step, and a full field measurement takes nearly $10 \mathrm{~min}$. A recorded $\mathrm{PHz}$ optical oscilloscope trace is shown in (b). We normalize the XUV intensity at each delay step, and find the peak of the XUV profile as a function of delay to yield the signal $\sigma(t)=\mathrm{d} E(t) / \mathrm{d} t$ (white line). We digitally filter the signal with a bandpass filter from $400 \mathrm{~nm}$ to $8 \mu \mathrm{m}$ and integrate to retrieve the field $E(t)$. In (c), we compare the PHz optical oscilloscope reconstructed field (red) to one retrieved with SALMON (blue), which was recorded in $100 \mathrm{~ms}$. Small differences in pulse shape are expected because of changes in CEP, the dispersion due to passing the signal field through the vacuum chamber window, and day-today changes in the laser conditions.

\subsection{Group delay dispersion (GDD) measurements}

As discussed in the theory section, as opposed to the $\mathrm{PHz}$ optical oscilloscope technique the retrieved field is nearly insensitive of the generating pulse shape. To emphasize this point, we insert fused silica glass into the generating beam path and measure the GDD. By placing $0 \mathrm{~mm}, 1 \mathrm{~mm}, 3 \mathrm{~mm}$, and $6 \mathrm{~mm}$ (blue, red, black, and green, respectively) of fused silica in the generating beam path, we observe little change to the retrieved field shapes, as shown in figure 5(a). It should be noted that for the 3 and $6 \mathrm{~mm}$ cases, the decreased generating beam peak intensity decreased the amount of third harmonic 


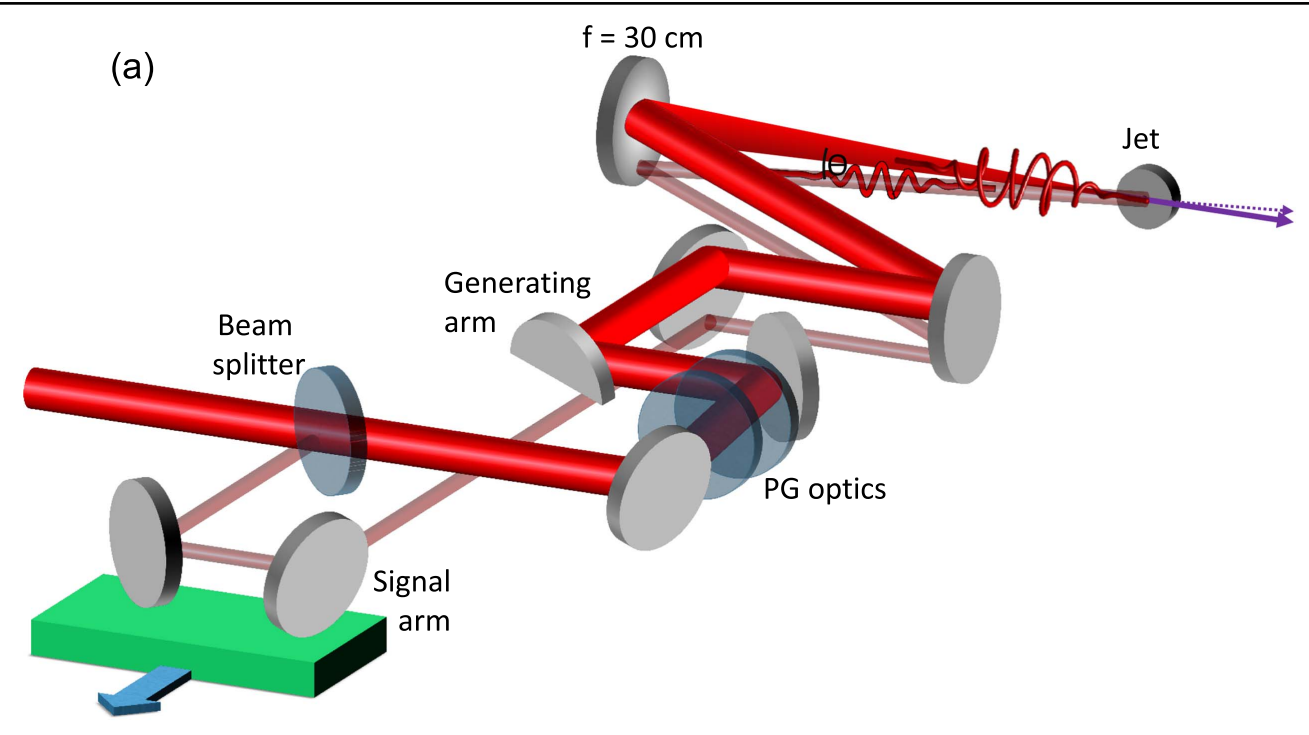

Delay stage
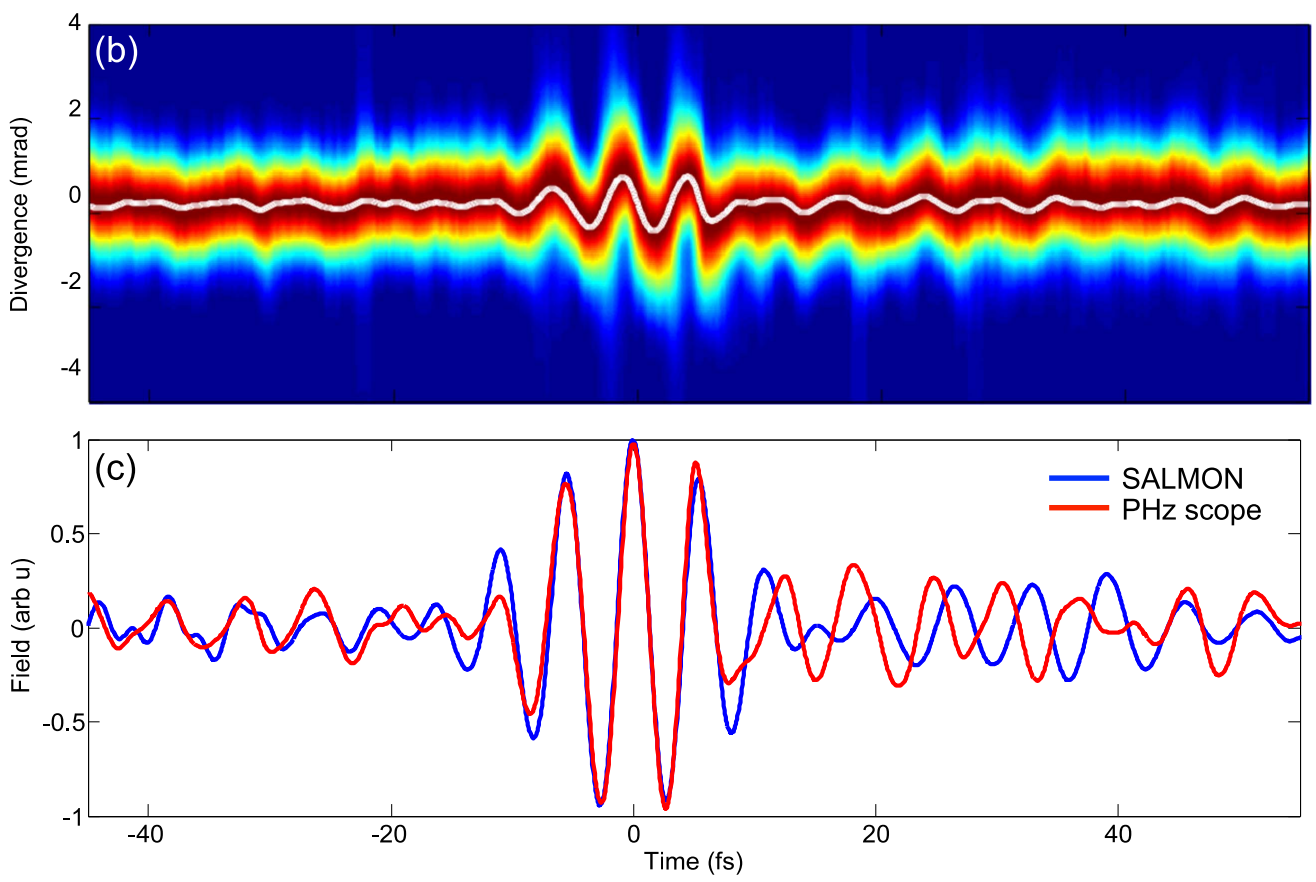

Figure 4. (a) Setup for PHz optical oscilloscope. The signal arm, with a small relative angle to the generating arm $\theta$, deflects the generated XUV direction (purple dash). PG polarization gating. (b) The measured PHz optical oscilloscope trace measuring the XUV deflection. (c) The PHz optical oscilloscope reconstructed field (red) compared to the SALMON measurement (blue).

generated. In order to maintain that the signal field is much weaker than the generating beam, we further decreased the signal intensity, increasing the noise.

Conversely, inserting glass into the signal beam path significantly changes the phase and increases the pulse duration of the measured field, as shown in figure 5(b). We place the same amount of fused silica in the signal beam path and observe an increase in pulse duration. We also observe that the pulse becomes significantly chirped with 3 and $6 \mathrm{~mm}$ fused silica.

We Fourier transform the measured fields to determine the spectral phase, shown in figures 5(c) and (d). We fit the retrieved spectral phases (solid) with a 3rd order polynomial fit (dashed). We add an arbitrary offset to the phase for visibility. In the case of inserting glass in the generating beam path (c), we observe little change to the spectral phase. However, we do observe a significant change in the spectral phase when the glass is inserted in the signal beam path, (d), as expected. We use the fits to take the second derivative to determine the GDD.

We retrieve the GDD, shown in figure 6. We fit the retrieved phase to a third-order polynomial and calculate the GDD at $1.61 \mu \mathrm{m}$. We find this slope $\left(-39 \mathrm{fs}^{2} \mathrm{~mm}^{-1}\right)$ is in good agreement with the dispersion calculated by the Sellmeier equation for fused silica at $1.64 \mu \mathrm{m}$, including an offset of $-21 \mathrm{fs}^{2}$, implying that the signal field experiences slight anomalous dispersion due to some optics before we make the SALMON measurement. We also measure the GDD at 

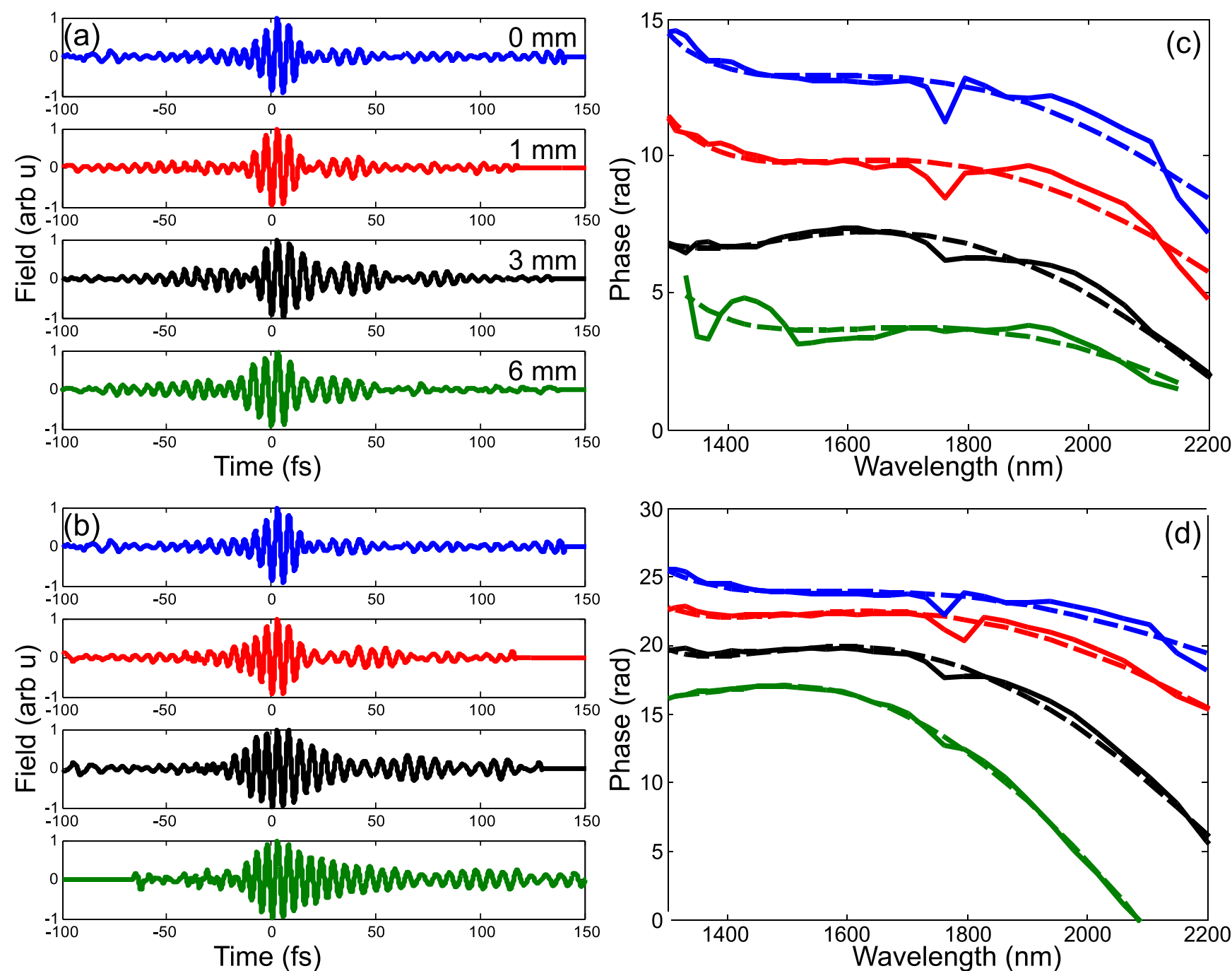

Figure 5. Retrieved signal fields when inserting $0 \mathrm{~mm}, 1 \mathrm{~mm}, 3 \mathrm{~mm}$, and $6 \mathrm{~mm}$ (blue, red, black, green, respectively) glass in the (a) generating beam path and (b) signal beam path. Label is inserted fused silica thickness. (c) Measured (solid) and fit (dashed) phases when inserting glass in the (c) generating and (d) signal beam paths.

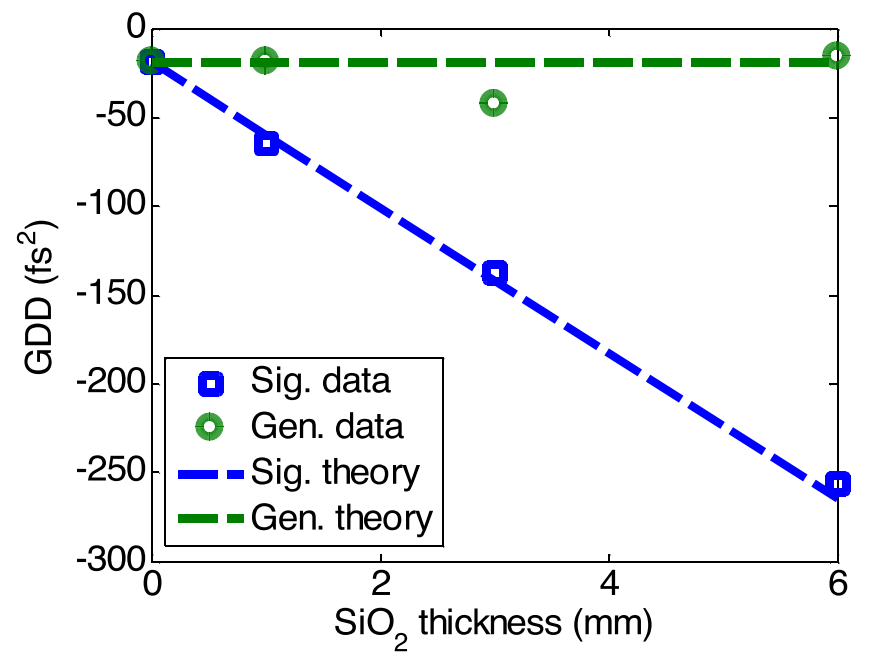

Figure 6. The measured dispersion in the signal field at $1.61 \mu \mathrm{m}$ by varying the amount of fused silica glass in signal (squares) and generating beam paths (circles). The signal theory is for dispersion at $1.64 \mu \mathrm{m}\left(-39 \mathrm{fs}^{2} \mathrm{~mm}^{-1}\right)$ with an offset of $-21 \mathrm{fs}^{2}$ (blue dash).
$1.8 \mu \mathrm{m}$ by fitting the data to a fourth order polynomial and find that the measured value of $-59 \mathrm{fs}^{2} \mathrm{~mm}^{-1}$ agrees well with the expected $-63 \mathrm{fs}^{2} \mathrm{~mm}^{-1}$.

\subsection{Polarization gating field retrieval}

We now measure a few-cycle pulse with rapidly varying polarization. One popular method for generating an isolated attosecond pulse is polarization gating [23-25]. This gating technique is easy to implement, requiring a birefringent quartz plate and quarter wave plate. The birefringent plate, with fast and slow axes at $45^{\circ}$ to the initially linearly polarized field, separates the few-cycle pulse into two orthogonally polarized pulses delayed in time by the optical path length difference caused by the difference in indices of refraction. For $1.8 \mu \mathrm{m}$ wavelength light, we can delay the two polarization states by $1.5 \lambda$ with $300 \mu \mathrm{m}$ of quartz. The quarter wave plate then creates two counter-rotating circularly polarized pulses with time-varying ellipticity; the two pulses interfere in the middle to create a single cycle linearly polarized field. 


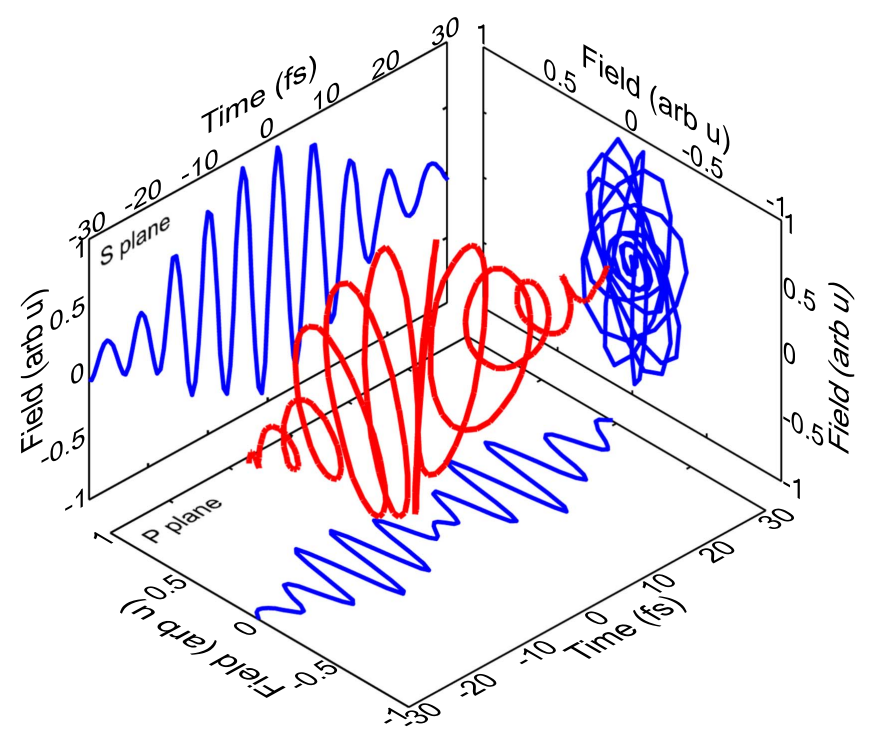

Figure 7. Measurement of polarization gating pulse. The S-plane shows the increased pulse duration while the P-plane shows the two few-cycle pulses, where the minimum is the gate. The 3D measured field (red) shows two counter-rotating fields.

In figure 7 , we show that we are able to measure a field with time-dependent polarization [26, 27]. The proper quartz thickness and orientation had been previously confirmed by observing the spectral modulation caused by the two interfering pulses. We place the polarization gating optics in the signal field path. In the generating beam path we place and rotate a half wave plate to project the polarization gated signal field on to the generating beam polarization. By rotating the half wave plate we are able to map out the rapidly changing polarization. In the P-plane, we observe the two few-cycle pulses while in the S-plane we observe a longer and larger amplitude pulse. The red curve is the three-dimensional reconstruction of the field. This experimentally measured field agrees well with the theoretically predicted field for polarization gating.

\subsection{Delay and CEP measurement}

The SALMON method is not sensitive to the global phase, only the relative phase of the generating and signal fields, and the phase is not sensitive to the relative delay of the two fields. We confirm these predictions in figure 8 . We delay the signal field using a nanometer resolution computer controlled delay stage over a range of $40 \mathrm{fs}$, shown in (a). Note that the change in the amplitude is due to normalizing the counts. We remove the Gaussian background to retrieve the field $S(\tau)$ in (b). We account for the stage delay in (c), showing that the retrieved field is independent of the relative delay and independent of the Gaussian generating beam shape.

Using a pair of thin fused silica wedges after the hollowcore fibre, we change the CEP of both signal and generating fields simultaneously. We note that there is no observed change in the measured field phase when changing the global CEP. Conversely, we are able to measure the CEP of the signal field relative to the generating beam by inserting a
$0.5 \mathrm{~mm}$ fused silica plate in the signal field path and tilting it. We predict a change of CEP by $\Delta \phi_{\mathrm{CEP}}=k z\left(n_{g}-n_{\phi}\right)$, where $k=2 \pi / \lambda, z$ is the length, and $n_{g}=1.4648$ and $n_{\phi}=1.4409$ are the group and phase indices of refraction for a central wavelength of $\lambda=1.8 \mu \mathrm{m}$. In this case, we get $z=75 \mu \mathrm{m}$, or a delay of $116 \mathrm{fs}$, for a CEP change of $2 \pi$.

We show that tilting the plate delays the signal field, moving the interference fringes over the generating beam profile in (d). Retrieving the field, (e), shows the delay of $190 \mathrm{fs}$ in $40^{\circ}$ of tilt. We account for the group delay to re-centre the pulse to show the phase change relative to the pulse envelope, which is the CEP, shown in (f). With SALMON, we are able to continually monitor the CEP evolution because of the large time window available and quick acquisition time.

\subsection{Intensity dependent sub-femtosecond phase shift}

Since we have established that the SALMON method can measure the relative phase of the generating and signal fields, we now measure a nonlinear phase shift induced by the high intensity few-cycle pulse. To this end, we focus the signal field using a $20 \mathrm{~cm}$ focal length mirror and re-collimate the beam using another $20 \mathrm{~cm}$ focal length mirror $40 \mathrm{~cm}$ away. We place an $80 \mu \mathrm{m}$ thick quartz sample on a translation stage, moving the sample through the focus using the SALMON method as a time-domain z-scan measurement. In this configuration, we are able to keep the intensity of the signal field constant for the field retrieval while continuously changing the intensity on the sample. The quartz optic axis is parallel to the polarization. The resulting measured fields are shown in figure 9 . The minimum intensity on the sample is estimated to be $0.5 \mathrm{TW} \mathrm{cm}^{-2}$ (blue) and the maximum is $10 \mathrm{TW} \mathrm{cm}^{-2}$ (red). As stated previously, we cannot distinguish interfering third harmonic; at higher intensities than those shown here, we generate significant third harmonic from the sample near the focus.

Interestingly, we measure the largest phase difference after the peak of the pulse. We ensure that the leading portion of the field shares the same phase, shown in (b), where the relative delay is within the uncertainty of the measurement. We define the delay by the zero crossing of the field. Near the peak of the pulse at $2 \mathrm{fs}$, the field is delayed by 350 as, (c). At $11 \mathrm{fs}$ after the peak of the field, we measure a maximum delay of 400 as, (d). The delay at the maximum of the field is approximately half of what we expect from the intensity and sample thickness.

The decreased phase delay is due to the propagation in free space of the Gaussian beam profile. We simulate the Gaussian beam propagation in quartz by calculating the nonlinear phase shift by the Forward Maxwell's equation [28], not accounting for non-perturbative effects such as ionization. We include the Gaussian profile intensity distribution, but assume that the quartz is thin enough such that the change in radial profile can be neglected within the quartz, that is $\nabla_{\perp}^{2} E(r)=0$. After passing through the quartz, we include the full $3 \mathrm{D}$ propagation for a few-cycle pulse using a Henkel transform to account for radial symmetry [29]. 

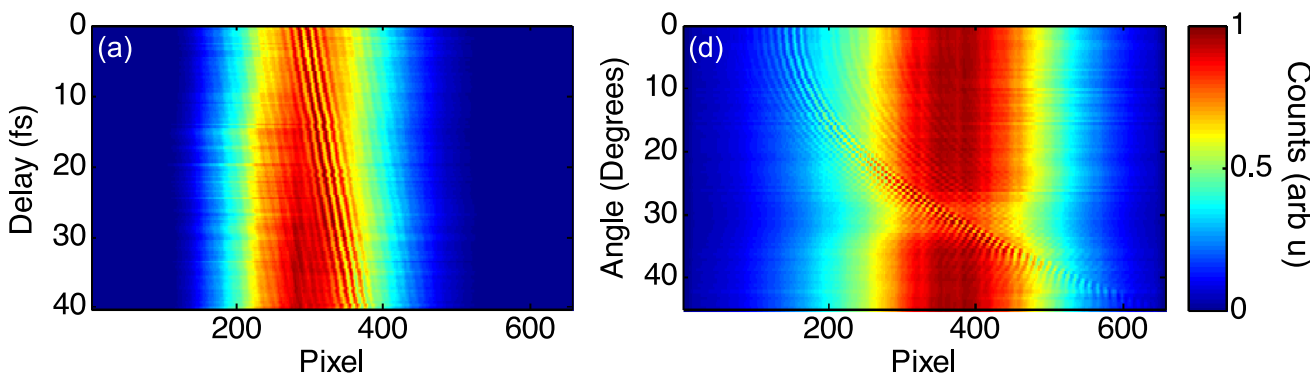

\section{西}
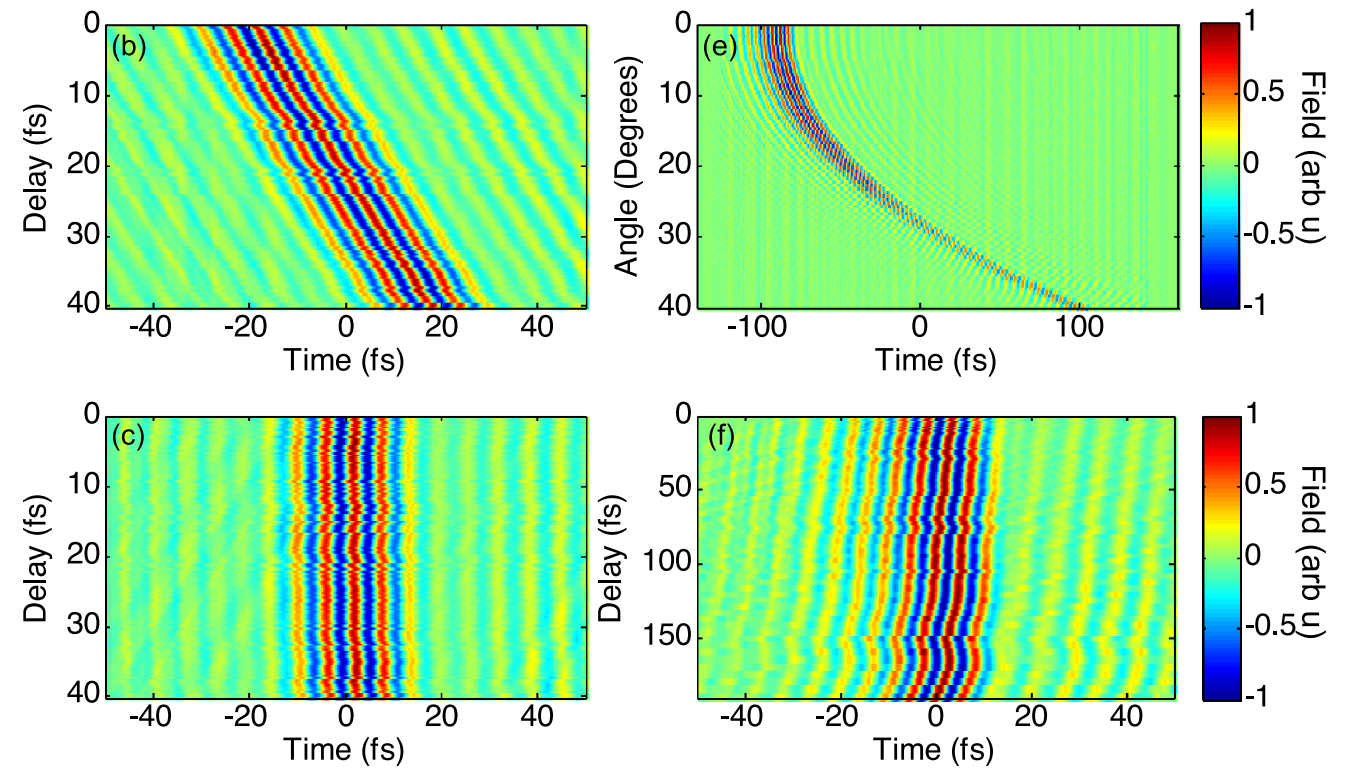

Figure 8. Left column: delay, and right column: CEP measurement of the two-cycle pulse. Top row: measured CCD counts across $x$ spatial coordinate; middle row: retrieved field $S(\tau)$; bottom row: field accounting for delays. The retrieved field is independent of the relative delay (c); we can measure the relative CEP caused by changing glass thickness (f).

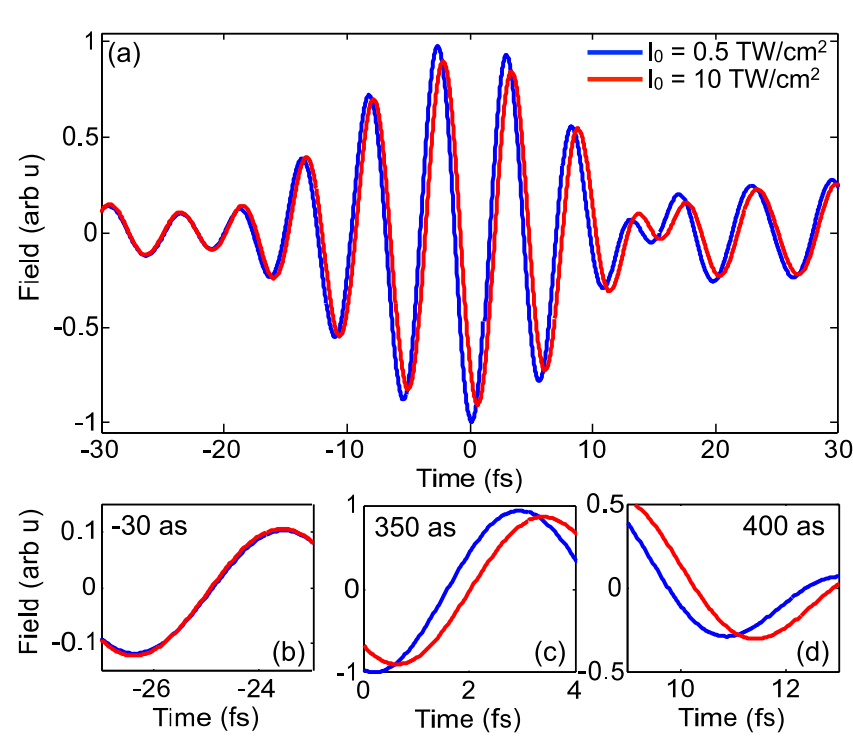

Figure 9. Time-dependent nonlinear phase shift measurement. We compare the retrieved field for low (blue) and high (red) intensities. The nonlinear interaction decreases the amplitude and delays the field about the peak of the pulse, and lasts for approximately $20 \mathrm{fs}$. Bottom row: magnification of the field (b) before the peak, (c) near the peak, and (d) after the peak of the pulse; delay is measured relative to zero crossing.
In figure 10, we plot the on-axis field from low $\left(I_{0}=\right.$ $\left.0.1 \mathrm{TW} \mathrm{cm}^{-2}\right)$ and high $\left(I_{0}=10 \mathrm{TW} \mathrm{cm}^{-2}\right)$ intensity cases, blue and red curves respectively. We simulate the experimental conditions of a Gaussian beam with waist $w_{0}=50 \mu \mathrm{m}, 15$ fs pulse duration centred at $1.8 \mu \mathrm{m}$. This beam passes through $80 \mu \mathrm{m}$ thick quartz, with polarization parallel to the optic axis. The intensity dependent phase shift causes self-focussing after the quartz plate. Immediately after the quartz plate, (a), we observe the intensity dependent phase delay between the high and low intensity cases on the propagation axis. The fields are normalised to their peak values. To demonstrate the effect of linear propagation on the delayed wavefront, we propagate the beam $1 \mathrm{~cm}$ after the quartz (b). In this case, the intensity dependent phase delay decreases. This result implies that time-dependent nonlinear phase shifts are sensitive to linear propagation effects, and that quantitative analysis must account for these effects [30].

Although the nonlinear phase shift is dependent on the peak intensity, we find that the pulse duration also affects the phase shift after the peak of the field has passed [31]. This delayed phase shift may be due to non-perturbative terms that come from real transitions to the conduction band that occur from multiple ionization events. In order to determine the dependence of these transitions on peak intensity, pulse duration, and central wavelength, we require a robust and 

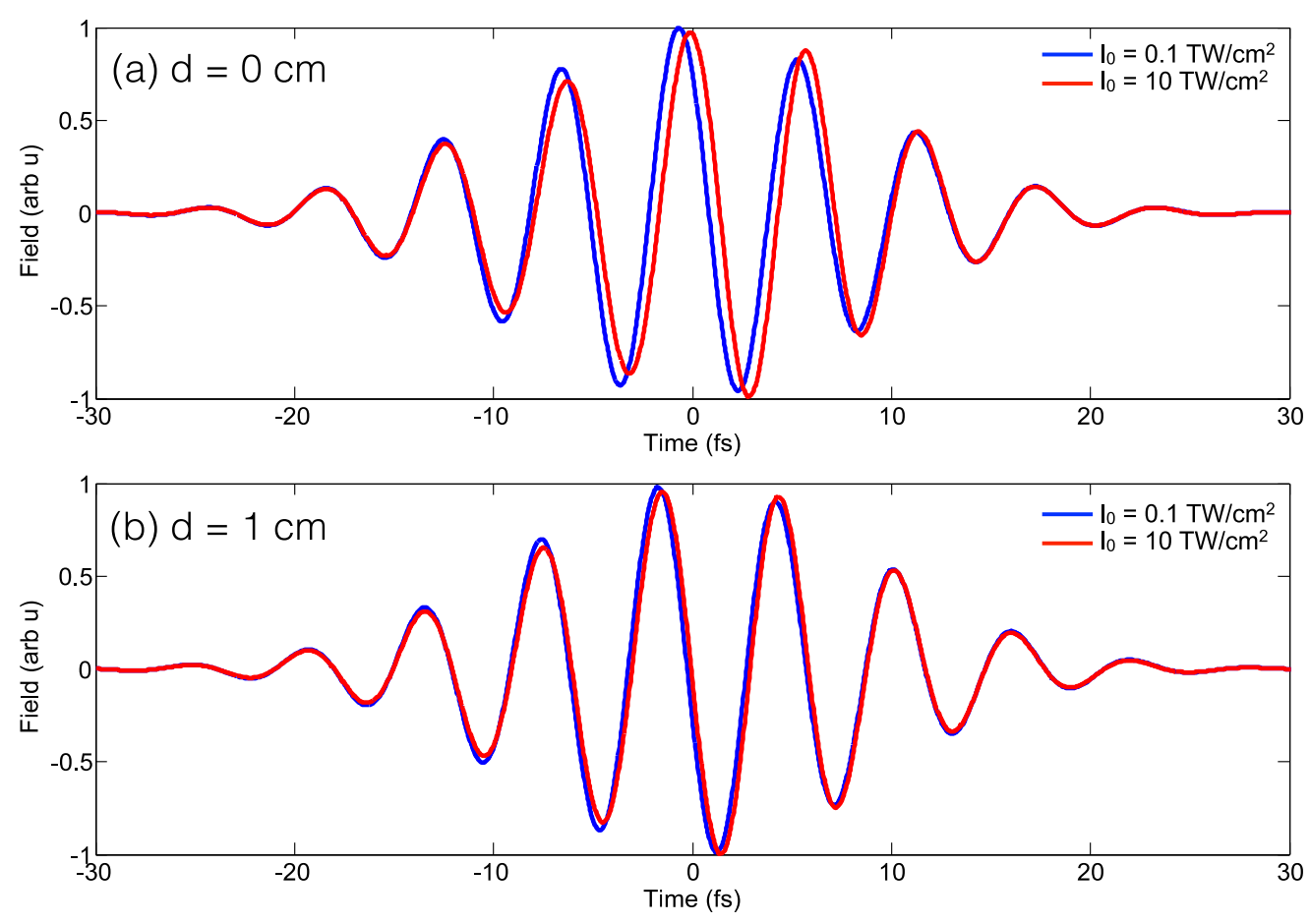

Figure 10. The propagation of a simulated Gaussian beam immediately after $80 \mu \mathrm{m}$ quartz (a) and after $1 \mathrm{~cm}$ linear propagation (b). Linear propagation decreases the intensity dependent phase shift.

quick method to measure these phase shifts. We predict that the SALMON technique will be able to efficiently explore these parameter spaces.

\section{Conclusion}

The SALMON method is a quick, accurate, and robust timedomain technique to measure the electric field for few-cycle laser pulses. For the measurement of two-cycle pulses we do not require moving parts, which allows for the real-time measurement and optimization of the field for compression of broadband spectra to transform-limited pulses. The measurement is sensitive to the field amplitude, and as such we can accurately measure the pre- and post-pulses that are common to few-cycle pulses, and with the large available temporal window we are able to easily reconstruct the spectrum and spectral phase. Additionally, because we are sensitive to the polarization we can measure rapidly varying elliptically polarized pulses, useful for attosecond science [32].

SALMON can be used as a complimentary technique to FTIR spectroscopy [33]. FTIR requires the field to be wellsampled in order to determine the carrier frequency, but the span must also be large for spectral resolution. With continuous sources, sampling the carrier frequency can be performed relatively quickly, but for low repetition rate sources this acquisition becomes increasingly tedious. With SALMON, the sub-cycle temporal resolution and the time window of hundreds of femtoseconds allows for picosecond time scans to be performed in very few shots, enabling rapid measurements of intensity dependent responses in the infrared.
In the measurement discussed here, we access one dimension of the generating beam profile and only reconstruct the signal field in time. However, there are several developed techniques to characterize the spatial dependence of few-cycle pulses [34-36]. We can modify the measurement to use both available dimensions to characterize the spatio-temporal dependence, for example pulses with pulse front tilt. SALMON will allow for the measurement of the spatial chirp and spectral phase as a spatio-temporal coupled pulse passes through the focus [37].

Although we only discuss a measurement of a signal field of similar bandwidth to the generating beam, we can measure signal fields outside of the generating beam bandwidth, as implied by figure 5(a). It may be possible to modify this technique to measure fields of wavelengths significantly longer than the generating pulse in the far-infrared and even terahertz regimes $[38,39]$.

In this experimental setup, we demonstrate the field reconstruction through the $\chi^{(3)}$ process in a nonlinear optic and image this interaction on the CCD detector. Because we are using a driving field in a spectral region where the camera sensor is insensitive we can use the CCD chip itself as the nonlinear optic [40, 41]. By imaging the nonlinearity directly on the camera we can avoid propagation effects. Furthermore, we may be able to measure a signal field that is significantly shorter in duration than the generating pulse through nonperturbative processes such as tunnel ionization on a microchannel plate (MCP), which is blind even in the visible. By increasing the detector nonlinearity order with the MCP, the effective pulse duration of the generating beam would then be strongly peaked about its field maximum. It may be possible to measure a signal field with a duration shorter than $1 \mathrm{fs}$, with 
frequency components well outside the generating pulse bandwidth.

\section{Acknowledgments}

We acknowledge the financial support from the US Army Research Office award number W911NF-14-1-0383 and US Air Force Office for Scientific Research award number FA9550-16-1-0109. TJH acknowledges financial support from AFOSR MURI grant number FA9550-15-1-0037.

\section{ORCID iDs}

TJ Hammond (iD https://orcid.org/0000-0001-8334-4332

D M Villeneuve (iD https://orcid.org/0000-0002-2810-3648

\section{References}

[1] Assion A, Baumert T, Bergt M, Brixner T, Kiefer B, Seyfried V, Strehle M and Gerber G 1998 Control of chemical reactions by feedback-optimized phase-shaped femtosecond laser pulses Science 282 919-22

[2] Silberberg Y 2009 Quantum coherent control for nonlinear spectroscopy and microscopy Annu. Rev. Phys. Chem. 60 277-92

[3] Joffre M, Hulin D, Migus A, Antonetti A, Benoit à la Guillaume C, Peyghambarian N, Lindberg M and Koch S W 1988 Coherent effects in pump-probe spectroscopy of excitons Opt. Lett. 13 276-8

[4] Woutersen S, Emmerichs U and Bakker H J 1997 Femtosecond mid-ir pump-probe spectroscopy of liquid water: evidence for a two-component structure Science 278 658-60

[5] Kampfrath T, Tanaka K and Nelson K A 2013 Resonant and nonresonant control over matter and light by intense terahertz transients Nat. Photon. 7 680-90

[6] Dorrer C, Belabas N, Likforman J-P and Joffre M 2000 Spectral resolution and sampling issues in Fourier-transform spectral interferometry J. Opt. Soc. Am. B 17 1795-802

[7] Griffiths P R and Haseth J A 2007 Fourier Transform Infrared Spectrometry (New York: Wiley)

[8] Bartels R, Backus S, Christov I, Kapteyn H and Murnane M 2001 Attosecond time-scale feedback control of coherent x-ray generation Chem. Phys. 267 277-89

[9] Lee D G, Kim J-H, Hong K-H and Nam C H 2001 Coherent control of high-order harmonics with chirped femtosecond laser pulses Phys. Rev. Lett. 87243902

[10] Schmidt B E, Shiner A D, Giguère M, Lassonde P, Trallero-Herrero C A, Kieffer J C, Corkum P B, Villeneuve D M and Légaré F 2012 High harmonic generation with long-wavelength few-cycle laser pulses J. Phys. B: At. Mol. Opt. Phys. 45074008

[11] Kim K T, Villeneuve D M and Corkum P B 2014 Manipulating quantum paths for novel attosecond measurement methods Nat. Photon. 8 187-94

[12] Goulielmakis E et al 2004 Direct measurement of light waves Science 3051267

[13] Mairesse Y and Quéré F 2005 Frequency-resolved optical gating for complete reconstruction of attosecond bursts Phys. Rev. A 71 011401(R)
[14] Kim K T, Zhang C, Shiner A D, Kirkwood S E, Frumker E, Gariepy G, Naumov A, Villeneuve D M and Corkum P B 2013 Manipulation of quantum paths for space-time characterization of attosecond pulses Nat. Phys. 9 159-63

[15] Salin F, Georges P, Roger G and Brun A 1987 Single-shot measurement of a 52 fs pulse Appl. Opt. 264531

[16] Tcherbakoff O, Mével E, Descamps D, Plumridge J and Constant E 2003 Time-gated high-order harmonic generation Phys. Rev. A 68043804

[17] Shan B, Ghimire S and Chang Z 2004 Generation of the attosecond extreme ultraviolet super continuum by a polarization gating J. Mod. Opt. 52 277-83

[18] Diels J-C M, Fontaine J J, McMichael I C and Simoni F 1985 Control and measurement of ultrashort pulse shapes (in amplitude and phase) with femtosecond accuracy Appl. Opt. 24 1270-82

[19] Trebino R 2000 Frequency-Resolved Optical Gating: The Measurement of Ultrashort Laser Pulses (Berlin: Springer)

[20] Walmsley I A and Dorrer C 2009 Characterization of ultrashort electromagnetic pulses Adv. Opt. Photonics 1308

[21] Kim K T, Zhang C, Shiner A D, Schmidt B E, Légaré F, Villeneuve D M and Corkum P B 2013 Petahertz optical oscilloscope Nat. Photon. 7 958-62

[22] Hammond T J, Villeneuve D M and Corkum P B 2017 Producing and controlling half-cycle near-infrared electricfield transients Optica 4 826-30

[23] Sola I J et al 2006 Controlling attosecond electron dynamics by phase-stabilized polarization gating Nat. Phys. 2 319-22

[24] Sansone G et al 2006 Isolated single-cycle attosecond pulses Science 314443

[25] Sansone G, Poletto L and Nisoli M 2011 High-energy attosecond light sources Nat. Photon. 5 655-63

[26] Lin M W and Jovanovic I 2016 Single-shot measurement of temporally-dependent polarization state of femtosecond pulses by angle-multiplexed spectral-spatial interferometry Sci. Rep. 632839

[27] Carpeggiani P et al 2017 Vectorial optical field reconstruction by attosecond spatial interferometry Nat. Photon. 11 383-9

[28] Husakou A V and Hermann J 2001 Supercontinuum generation of higher-order solitons by fission in photonic crystal fibers Phys. Rev. Lett. 87203901

[29] Hammond T J, Monchocé S, Zhang C, Vampa G, Klug D, Naumov A Y, Villeneuve D M and Corkum P B 2017 Integrating solids and gases for attosecond pulse generation Nat. Photon. 11 594-9

[30] Sommer A et al 2016 Attosecond nonlinear polarization and light-matter energy transfer in solids Nature 534 86-90

[31] McDonald C R, Vampa G, Corkum P B and Brabec T 2017 Intense-laser solid state physics: unraveling the difference between semiconductors and dielectrics Phys. Rev. Lett. 118 173601

[32] Cunningham E and Chang Z 2015 Optical gating with asymmetric field ratios for isolated attosecond pulse generation J. Sel. Top. Quantum Electron. 211

[33] Lepetit L, Chériaux G and Joffre M 1995 Linear techniques of phase measurement by femtosecond spectral interferometry for applications in spectroscopy J. Opt. Soc. Am. B 12 2467-74

[34] Gabolde P and Trebino R 2008 Single-frame measurement of the complete spatiotemporal intensity and phase of ultrashort laser pulses using wavelength-multiplexed digital holography J. Opt. Soc. Am. B 25 A25

[35] Miranda M, Kotur M, Rudawski P, Guo C, Harth A, L'Huillier A and Arnold C L 2014 Spatiotemporal characterization of ultrashort laser pulses using spatially resolved fourier transform spectrometry Opt. Lett. 39 $5142-5$ 
[36] Parent G, Gallet V, Borot A, Gobert O and Quéré F 2016 Space-time characterization of ultra-intense femtosecond laser beams Nat. Photon. 10 547-53

[37] Hammond T J, Balogh E and Kim K T 2017 Isolation of attosecond pulses from the attosecond lighthouse J. Phys. B: At. Mol. Opt. Phys. 50014006

[38] Jamison S P, Shen J, MacLeod A M, Gillespie W A and Jaroszynski D A 2003 High-temporal-resolution, single-shot characterization of terahertz pulses Opt. Lett. 281712
[39] Keiber S, Sederberg S, Schwarz A, Trubetskov M, Pervak V, Krausz F and Karpowicz N 2016 Electro-optic sampling of near-infrared waveforms Nat. Photon. 10159

[40] Padgett M J, Harvey A R, Duncan A J and Sibbett W 1994 Single-pulse, Fourier-transform spectrometer having no moving parts Appl. Opt. 336035

[41] Kelly T J, Davitt S J and Costello J T 2016 A simple, low cost interferometric autocorrelator with no moving parts Meas. Sci. Technol. 27117003 